Chapman University

Chapman University Digital Commons

Pharmacy Faculty Articles and Research

School of Pharmacy

2014

\title{
Nanoparticulate Drug Delivery Platforms for Advancing Bone Infection Therapies
}

Vuk Uskoković

ChapmanUniversity, uskokovi@chapman.edu

Tejal A. Dasai

University of California - San Francisco

Follow this and additional works at: http://digitalcommons.chapman.edu/pharmacy_articles

Part of the Bacterial Infections and Mycoses Commons, Nanomedicine Commons, Other Pharmacy and Pharmaceutical Sciences Commons, and the Pharmaceutics and Drug Design Commons

\section{Recommended Citation}

Uskoković V, Desai TA. Nanoparticulate drug delivery platforms for advancing bone infection therapies. Expert Opin Drug Deliv. 2014;11(12):1899-1912. doi:10.1517/17425247.2014.944860.

This Article is brought to you for free and open access by the School of Pharmacy at Chapman University Digital Commons. It has been accepted for inclusion in Pharmacy Faculty Articles and Research by an authorized administrator of Chapman University Digital Commons. For more information,

please contact laughtin@chapman.edu. 


\section{Nanoparticulate Drug Delivery Platforms for Advancing Bone Infection Therapies}

\section{Comments}

This is an Accepted Manuscript of an article published in Expert Opinion on Drug Delivery, volume 11, issue 12, in 2014, available online: http://www.tandfonline.com/10.1517/17425247.2014.944860.

\section{Copyright}

Taylor \& Francis 


\title{
Nanoparticulate drug delivery platforms for advancing bone infection therapies
}

\author{
Vuk Uskoković ${ }^{1, \dagger}$ and Tejal A Desai ${ }^{2,{ }^{*}}$ \\ ${ }^{1}$ University of Illinois, Department of Bioengineering, Advanced Materials and Bionanotechnology \\ Laboratory, Chicago, IL 60607-7052, USA \\ 2University of California, Therapeutic Micro and Nanotechnology Laboratory, Department of \\ Bioengineering and Therapeutic Sciences, San Francisco, CA 94158-2330, USA
}

\begin{abstract}
Introduction-The ongoing surge of resistance of bacterial pathogens to antibiotic therapies and the consistently aging median member of the human race signal an impending increase in the incidence of chronic bone infection. Nanotechnological platforms for local and sustained delivery of therapeutics hold the greatest potential for providing minimally invasive and maximally regenerative therapies for this rare but persistent condition.
\end{abstract}

Areas covered-Shortcomings of the clinically available treatment options, including poly(methyl methacrylate) beads and calcium sulfate cements, are discussed and their transcending using calcium-phosphate/polymeric nanoparticulate composites is foreseen. Bone is a composite wherein the weakness of each component alone is compensated for by the strength of its complement and an ideal bone substitute should be fundamentally the same.

Expert opinion-Discrepancy between in vitro and in vivo bioactivity assessments is highlighted, alongside the inherent imperfectness of the former. Challenges entailing the crossdisciplinary nature of engineering a new generation of drug delivery vehicles are delineated and it is concluded that the future for the nanoparticulate therapeutic carriers belongs to multifunctional, synergistic and theranostic composites capable of simultaneously targeting, monitoring and treating internal organismic disturbances in a smart, feedback fashion and in direct response to the demands of the local environment.

\section{Keywords}

bone; calcium phosphate; composite; infectious disease; nanoparticle; osteomyelitis; tissue engineering

\footnotetext{
(C) 2014 Informa UK, Ltd.

†, ${ }^{*}$ Authors for correspondence: University of Illinois, Department of Bioengineering, Advanced Materials and Bionanotechnology Laboratory, Chicago, IL, USA, uskok@uic.edu, tejal.desai@ucsf.edu.

Declaration of interest

The authors report no conflicts of interest. The authors alone are responsible for the content and writing of the paper.
} 


\section{Introduction}

Since the advent of the antibiotic era, infectious diseases have lost a place at the forefront of interest of biomedical research in the developed world. For many decades now, with the exception of HIV, the concern for contracting a major infection among individuals with uncompromised immune responses has been confined to the Third World countries. Not only does the overall National Institutes of Health funding for infectious diseases and their clinical corollaries currently comprise mere $10 \%$ of its overall amount, but the funding for rural health in 2014 has, for example, outstripped that for malaria by $>50 \%$ [1]. However, with the evolution of bacterial resistance to the current generation of antibiotics and the corresponding proneness to latent viral outbreaks, this state of affairs is expected to change in the near future. Finding the treatment for the hyperaggressive forms of bacterial infections before the humanity witnesses an impending pandemic of devastating proportions proves as necessary and might, moreover, require a fundamentally different therapeutic approach compared to the one that has been embraced in the past.

Bone is an organ shielded well from the external pathogens, which is a double-edged sword from the clinical standpoint. For, to the same extent that it resists infection, it resists therapies against the occurring infections. Despite the extensive research, osteomyelitis proves difficult to treat and its chronic forms usually entail poor prognosis for the patient [2]. The difficulty of its treatment is further increased due to the fact that a wide variety of pathogens can cause it, including fungus, pox viruses as well as not so common bacteria, such as Haemophilus influenzae, Brucella suis, Mycobacterium tuberculosis, Mycobacterium ulcerans, Fusobacterium nucleatum and others, aside from the pyogenic bacteria from the healthy oral flora, which remain its main causative agents. Staphylococcus aureus and Staphylococcus epidermis are held responsible for the great majority of bone infections, $\sim 90 \%$, whereby the increasing incidence of their methicillin-resistant forms explains the unusual severity of the recent clinical manifestations of this disease characterized by a high recurrence rate despite the extensive surgical interventions and longterm antibiotic therapies [3].

Historically, given that osteomyelitis is one of the oldest documented diseases, whose first reports date back to Hippo-crates' scripts from the 5th century BC [4] and whose oldest known evidence lies in the fossilized spine of a 270 million-year-old Permian reptile [5], significant advances in its treatment have been made in the past [6]. Before the introduction of penicillin in the 1940s, chronic osteomyelitis was treated first by amputation, then by trepanning and less-invasive surgeries [7] with or without the application of various natural medicines, including such ointments as bovine feces or larva of the blow fly [8]. Mainstay of therapy for the last 50 years has, however, been a concoction of oral and parenteral antibiotic therapy, lasting up to $2-12$ weeks, depending on the severity of the infection, and surgical debridement of necrotic bone. The room for the improvement of this traditional, yet rather deficient, approach to the treatment of osteomyelitis is, however, obvious to those familiar with the sophisticated drug delivery technologies currently existing in the embryonic or translational stages (Figure 1). Its elaboration from a bionanotechnological perspective presents the subject of this opinion piece. 


\section{Shortcomings of the traditional therapies and the available local release alternatives}

Two of the major downsides of the traditional therapy for osteomyelitis are fairly obvious. First, the surgical removal of diseased bone entails weakening of the musculoskeletal support and causes unaesthetic disfigurements, frequently demanding the implantation of permanent braces besides bone grafting. Second, antibiotics administered systemically have a hard time crossing the sequestra (and the relatively avascular sclerotic bone surrounding it) and reaching the infection site, which does not only diminish their effectiveness, but also increases the chances for the induction of resistance of the pathogen to the actual antibiotic therapy. Logically, these two central shortcomings could be ameliorated with the use of platforms capable of locally and sustainably delivering antimicrobial agents while stimulating tissue regeneration and substitution of necrotic foci with the newly formed, healthy bone.

At the clinical level, these innovative strategies remain only partially implemented and that mainly in the form of poly (methyl methacrylate) (PMMA) beads. After they had been first clinically applied in the early 1970s [9], they gradually established themselves as a standard option for the local delivery of antibiotics to bone cavities and this trend continues to this very day. Although PMMA beads loaded with hydrophilic antibiotics were successfully applied in the past [10-12], numerous clinical limitations are associated with their use. These include: i) their nonbiodegradable nature and the need for a secondary surgical procedure to ensure their removal from the body after the release process is over [13]; ii) an often insubstantial amount of the released antibiotic following the initial, burst release phase [14], which has led to the promotion of pathogenic resistance to such therapies in the past [15]; iii) proneness to biofilm formation, which hinders the antimicrobial action [16]; and iv) moderate toxicity resulting from the absorption of MMA monomers and the carboxylesterase-mediated conversion of MMA to methacrylic acid [17]. On top of this, a comprehensive clinical study has yet to prove that PMMA beads are more effective than the systemic antibiotic delivery in treating orthopedic infections [18]. The major clinically available alternative capable of sustained release are calcium sulfate cements, which suffer from other weaknesses, mainly their rapid degradation time scale, in the order of weeks, which is faster than the bone ingrowth rate and can lead to the mechanical implant failure [19], alongside other clinical issues, such as drainage at the wound site [20], severe inflammation secondary to overabundance of calcium ions [21], and the occurrence of fibrous gaps in the zones undergoing the replacement of the rapidly resorbing cement with the slowly growing bone [22]. However, with the current knowledge of sophisticated drug delivery vehicles, devising a new generation of locally targeted and minimally invasive therapies for osteomyelitis could be readily foreseen.

\section{Biodegradable poly(a-hydroxy esters): pros outweighed by cons}

Biodegradable polymeric carriers, for example, pose themselves as a natural alternative to the use of PMMA. To this end, the favorite choice has fallen on poly(a-hydroxy esters), including poly(L-lactic acid) (PLLA), poly(glycolide) (PGA) and poly-(D,L-lactide-coglycolide) (PLGA), all of which have been experimentally applied as antibiotic carriers for 
the treatment of osteomyelitis [23-25]. An immediate upside of their application comes from their ability to encapsulate comparatively large amounts of both hydrophobic and hydrophilic drugs [26] and exhibit extended drug release profiles [27]. The latter can be furthermore made tunable via control over composition and processing conditions [28]. In the case of PLGA, for example, the lactide-to-co-glycolide ratio could be used as a control parameter in setting the degradation and release time scales under physiological conditions to range from a year or more for PLLA to around 6 months for PLGA with the lactide-to-coglycolide ratio of 1:1 to about a month for pure PGA. A detrimental effect that entails the use of poly (a-hydroxy esters) and that was observed in the past is that their acidic degradation products may favor the bacterial growth and promote hard tissue resorption and bone mass loss [29,30]. Chronic inflammation is, in fact, known to often result as a response to implantation of PLLA-based polymers in bone tissue engineering [31-33]. Since the antimicrobial effectiveness of antibiotics is found only within a relatively narrow window of $\mathrm{pH}$ values and could decrease by as much as 16-fold following a $\mathrm{pH}$ drop from 7.4 to 5.5 [34], this acidification entailing partial or complete degradation of poly (a-hydroxy esters) can negatively interfere with the antimicrobial action. Despite their superior release properties, this has suggested the necessity of combining poly(a-hydroxy esters) with alkaline inorganics, such as calcium phosphates [35].

\section{Calcium phosphates}

Calcium phosphates have been traditionally considered as a favorite choice for the synthetic substitute of hard tissues due to their structural and compositional versatility, outstanding biocompatibility and bioactivity, and the ability of being conducive to new bone growth. The major weakness of this material springs from the fact that it resists encapsulation of drugs via intercalation. Calcium phosphates are, on the other hand, excellent adsorbents of an array of organics, the reason for which their least-soluble phase, hydroxyapatite, has been used as a chromatographic adsorbent of proteins and nucleic acids [36,37]. This property of theirs, however, comes at a cost because it predisposes them in turn to exhibit burst release and puts definite limits on their capacity to release substantial amounts of drugs over prolonged periods of time. Although some level of burst release is desired to some extent in the treatment of infectious diseases, lest the targeted bacterial populations become potentially resistant to the administered antibiotic, the release from calcium phosphates is typically such that $80-90 \%$ of the drug becomes desorbed in the first minutes of contact with the physiological solution or body fluids [38]. The simplest way to overcome this issue has involved intraporous loading of nanoparticulate aggregates with a drug either using high pressure [39,40] or spontaneous powder compaction and drug capture upon desiccation [41]. These strategies, however, interfere with the attempts to produce injectable colloidal calcium phosphate gels and question the veracity of their nanoparticulate nature if they come to be applicable as solely implantable microscopic aggregates of nanoparticles.

Calcium phosphates belong to structurally complex ceramics wherein the stoichiometric composition could be used to tailor the properties of interest. Figure $2 \mathrm{~A}$ illustrates the use of another ceramic compound, lanthanum-strontium manganite, in producing tunable and selfregulating superparamagnetic materials for the treatment of cancer via hyperthermia. Namely, by varying the ratio between lanthanum and strontium, that is, parameter $x$ in 
nanosized $\mathrm{La}_{1-\mathrm{x}} \mathrm{Sr}_{\mathrm{x}} \mathrm{MnO}_{3}{ }_{\delta}$, the Neel point of the material changes in the range of $\mathrm{T}=25-$ $100^{\circ} \mathrm{C}$ for $\mathrm{x}=0.16-0.5$, enabling the use of the material for hyperthermic, thermoblastic or even more intense thermal tissue healing applications [42]. Similarly, calcium phosphates can be synthesized in a variety of stoichiometries, covering the range of solubility values from $18 \mathrm{~g} / \mathrm{dm}^{3}$ for anhydrous and monohydrous monocalcium phosphates (triclinic $\mathrm{Ca}\left(\mathrm{H}_{2} \mathrm{PO}_{4}\right)_{2}$ ) to $88 \mathrm{mg} / \mathrm{dm}^{3}$ for brushite (monoclinic $\mathrm{CaHPO}_{4} \cdot 2 \mathrm{H}_{2} \mathrm{O}$ ) to $48 \mathrm{mg} / \mathrm{dm}^{3}$ for monetite (triclinic $\mathrm{CaHPO}_{4}$ ) to $17 \mathrm{mg} / \mathrm{dm}^{3}$ for calcium pyrophosphate (tetragonal $\mathrm{Ca}_{2} \mathrm{P}_{2} \mathrm{O}_{7}$ ) to $2.5 \mathrm{mg} / \mathrm{dm}^{3}$ for a-tricalcium phosphate (monoclinic $\mathrm{Ca}_{3}\left(\mathrm{PO}_{4}\right)_{2}$ ) to $\sim 1 \mathrm{mg} / \mathrm{dm}^{3}$ for amorphous calcium phosphate to $0.3 \mathrm{mg} / \mathrm{dm}^{3}$ for hydroxy-apatite (pseudo-hexagonal $\left.\mathrm{Ca}_{10}\left(\mathrm{PO}_{4}\right)_{6}(\mathrm{OH})_{2}\right)$. In such a manner, the composition could be a control parameter for the solubility, that is, the degradation rate, which could be, in turn, used as a control parameter for the drug release. Figure 2B correspondingly demonstrates the use of different calcium phosphate phases for achieving different rates of release of bovine serum albumin [43].

Another ceramic material offering similar tailoring of the degradation rate to a desired kinetic profile via control of its stoichiometry is bioactive glass, which has also been researched extensively as a carrier for the sustained release of antibiotics in the treatment of orthopedic infections [44]. Such adjustments of the degradation rate could be achieved, for example, by controlling silicate-to-borate ratio [45] or the amount of a less-abundant constitutive ion, such as sodium [46] or strontium [47]. Still, given the fact that silicon, the main cationic component of all types of bioactive glasses, is but a trace element in the human body, present in the amount of only $1-2 \mathrm{~g}$ [48], as well as that their amorphous network of silica tetrahedra partially, yet usually incompletely [49], transforms to hydroxyapatite in the body raises fundamental issues regarding their usability as bone substitutes, especially when compared with a more natural compound of choice: calcium phosphate.

Being the sole component of the mineral phase of hard tissues, calcium phosphates are natural candidates for bone-filling drug carriers. With bone acting as a natural reservoir for calcium and phosphate ions [50], any excessive amounts thereof could be regulated in favor of new bone growth. Calcium and phosphate ions released upon degradation stimulate osteoblastic differentiation [51,52] and proliferation [53] and could be used as ionic ingredients for the new bone formation. Another advantage of calcium phosphates is that they could be readily made into self-setting pastes, injectable upon mixing, yet setting into firm solids after the injection [54]. Unlike polymers, calcium phosphates tolerate well the exposition to $\gamma$-irradiation, gas plasma discharge and supercritical $\mathrm{CO}_{2}$ and could be sterilized using any of those without any concerns that their structural properties would be damaged thereby. Calcium phosphate particles could be also fabricated in a variety of morphologies and nanosize presents their natural, most easily obtainable state by precipitation from aqueous solutions [55].

\section{The biomimetic argument}

Biomimetics, it is worth noticing, is one of the most prospective principles that guide tissue engineers in designing a new generation of biomaterials [56,57]. In this case, it can be summed as similia similibus curantur, necessitating the substitution of like with like. First, 
because bone is an organic/inorganic composite, it could be argued that a composite of a similar nature is to be used as its ideal substitute. Second, bone is a nanostructured composite, given that at the molecular level it comprises $\sim 30 \times 20 \times 2 \mathrm{~nm}$-sized calcium phosphate crystals interspersed within the collagen matrix [58]. Third, we have seen that calcium phosphate as the key inorganic ingredient of bone suggests the ideality of its usage as a core nanostructured component of composites for bone regeneration. Finally, bone is a multifunctional biological material involved in biomechanical support and protection of the body, production of blood cells and mineral ion storage. Therefore, it is natural to expect that the ideal material for its replacement should also be multifunctional. This is where we arrive once again at the simultaneously antimicrobial and regenerative role for the new generation of drug delivery platforms for the prophylactic and/or restorative treatments of bone affected by osteomyelitis. This idea is logical in view of the dual purpose that an ideal therapeutic agent is to serve in general: i) to eradicate the internal manifestation of illness; and ii) to revitalize the organism.

Biological techniques applicable in the assessment of the regenerative capacities of these platforms can be divided to morphological, genetic, proteomic and metabolic ones and are illustrated in Figure 3. Immunohistochemistry presents a valuable tool in understanding the osteoconductivity of materials by measuring the affinity of the cell to the surface of the carrier, as shown in Figure 3A. Expression of osteogenic markers at the mRNA level, including, most notably and least ambiguously, osteocalcin, Runx2 and osterix, could give an insight into the bone-building activity of osteoblasts in contact with the material (Figure 3B). The expression of proteins implicated in bone mineralization can be quantified to conclude about the osteogenic potency of the material (Figure 3C), while smaller cytokines and metabolites involved in signaling between osteoblasts and osteoclasts, the two types of cells that in a mutually antagonistic manner orchestrate the bone regeneration process, could be equally reliable indicators of the osteogenic response of the cells to a given material. Such molecules include members of the osteoprotegerin/receptor for activation of NF-kB ligand (RANKL)/RANK pathway, where RANKL is a tumor necrosis factor family member secreted by osteoblasts with a role to trigger the differentiation of mesenchymal stromal cells to osteoclasts and speed up the complementary bone degradation processes. Finally, the measurements of calcitriol, the active form of vitamin D, or retinoic acid (RA), both of which are crucial for the proper skeletal development, could be indicative of the osteogenic process, owing to the fact that their concentration increase corresponds to the upregulation of protein species in the osteogenic pathway, directly in the case of calcitriol [59] and inversely in the case of RA [60,61]. Figure 3D and E shows chromatograms of vitamin D metabolites extracted from a human sample using an antibody binding specifically to $1,25-$ dihydroxycholecalciferol [62] and a direct proportionality between its concentration at the cellular level and the osteogenic activity of the cells [63].

\section{Targeting the intracellular colonies}

The antibiotic treatments of osteomyelitis are greatly hampered due to the ability of $S$. aureus, its main causative agent, to penetrate osteoblastic and other cells and form a temporary symbiosis with them $[64,65]$, before inducing their apoptosis and continuing to spread across the organism [66]. Though far more difficult to achieve, targeting these 
intracellular colonies by the antibiotic therapy is thus expected to be more important for preventing the recurrence of infection than eradicating only the pathogens that colonize the bone matrix [67]. Calcium phosphate nanoparticles are the major nonviral agents for the intracellular delivery of plasmids [68,69], which explains the fact that, loaded with clindamycin phosphate, they diminished the presence of $S$. aureus colonies located within osteoblastic cells in vitro to a greater degree than it was achieved by the application of the pure antibiotic [70]. Figure 4A shows a schematic description of the nanoparticle uptake by the cell, while Figure 4B demonstrates the intracellular localization of calcium phosphate nanoparticles observed during an immunofluorescent analysis of their interface with osteoblastic cells.

\section{The prospect of composites}

The aforementioned arguments speak in favor of composite nanoparticles as the most prospective ones in ensuring simultaneously therapeutic and regenerative performance. Although increased bioactivity usually comes at the cost of increased proneness to biofilm formation, an optimal balance between the antibacterial (therapeutic) and the osteogenic (regenerative) performance of biomaterials designed to cope with bone infection must be found. And the fact that multiple functionalities are to be achieved by these materials points in the direction of composites as the forthcoming generation of materials for bone engineering. In addition, bone itself is a composite wherein the weakness of each component alone is compensated for by the strength of its complement and it could be argued that an ideal bone substitute should be fundamentally similar in nature. Polymeric coatings are capable of stabilizing the drug adsorbed on the surface of calcium phosphate nanoparticles and serving as an additional reservoir for drug encapsulation and its more sustained release [71], alongside offering a more stable surface for conjugation with targeting, optically active or other therapeutic ligands [72]. An alternative concept employs a nanoparticulate, microporous calcium phosphate shell formed around the polymeric core [73]. This reverse method aims to harness the osteoconductive nature of the ceramic phase to a greater extent, while still enabling the drug to be released sustainably from the core polymeric reservoir through the surface pores.

From the mechanical standpoint, it is generally assumed that the microstructure of calcium phosphates alone cannot be modified in such a manner as to make the material mechanically compatible with the grafted bone and eliminate the risk of filler fracture after its surgical placement [74]. Only in combination with a soft component could calcium phosphates overcome these fundamental problems associated with their clinical application. For example, the combination of visco-elastic polymers and osteoconductive calcium phosphates has yielded composites that surpassed the resistance to fracture, the structural integrity and the stiffness of individual components [75], making up for the low compressive strength of the former and brittleness and the lack of malleability of the latter [76]. Finally, the resorption time and the corresponding bone ingrowth rate significantly increased when hydroxyapatite was implanted as a bone substitute in vivo in a composite form, in combination with PLGA [77,78], a polymer known to be able to accelerate the resorption of calcium phosphates by the release of its acidic degradation products [79]. 
Of course, sky is the limit when it comes to conceiving additional components of such basic soft/hard organic/inorganic matrices that crudely mimic bone. Combinations of calcium phosphate, PLGA and semiconductor quantum dots at the nanoparticle scale have, for instance, enabled monitoring of the particle routes in the body [80,81]. Many of the readily imaginable options are, however, yet to be explored. Conjugation of the carrier particles to moieties that have an affinity for various bone components [82], such as bisphosphonates [83] or estrogen [84], acting as either targeting agents or metabologens, is one and the enrichment of antibiotic carriers with pluripotent cells, such as mesenchymal stem cells able to differentiate into osteoblasts [85], is another. Supplementation of particles with elements or surfaces with pronounced antimicrobial activities as alternatives to traditional antibiotics, always prone to induce the globally spreading resistance of pathogens to them, presents another prosperous direction of research. Shown in Figure 5 is an example of monodisperse, composite and antibiotic-free nanoparticles with simultaneously antioxidative, antibacterial and osteoinductive properties. The particles were able to: i) reduce the concentration of superoxide in human umbilical vein endothelial cells; ii) suppress the growth of E. coli and methicillin-resistant $S$. aureus; and iii) upregulate the expression of two osteogenic markers: osteocalcin and protocollagen type I.

\section{Expert opinion}

So far we have described the discrepancy between the therapeutic state of the art in coping with the cases of osteomyelitis in the clinic and the existing drug delivery technologies. In broader terms, a new set of regulations may be needed to facilitate the translational path between the bench and the clinic, lest this gap continues to widen. Also, enabling a greater flux of ideas from materials science centers of thought to the world of biology and medicine will be of benefit for the process of discovery of advanced nanoscale drug delivery platforms. One way to achieve this is to stimulate the opening of materials science centers in the core of medical school environments and convert physical proximity to fruitful collaboration around specific clinical priorities. Engineering a new generation of drug delivery vehicles is, after all, a discipline at a crossroads, requiring an input from the materials scientists, physical chemists, mechanical engineers, pharmacologists, molecular and cell biologists and, crucially, clinicians. How to balance the congregation of such a multitude of outlooks that often sound foreign to each other with maintaining an in-depth perspective toward a given therapeutic goal is a challenge for the new generation of principal investigators. In the end, with the subjects of research reflecting their qualities back to researchers, it comes as natural that a new generation of materials will raise a new generation of materials scientists developing them.

More specifically, an ideal material for bone infection therapies would be an injectable colloid capable of adhering to the defected bone mass, possibly through a self-setting mechanism. It would then proceed to release the antimicrobial pay-load to the areas of infection in a sustained and targeted manner, thwarting its progress while also being able to foster the resorption of the necrotic bone and its replacement with the newly grown one. The abovementioned similia similibus curantur maxim has guided us in the direction of calcium phosphates as ideal core components of complex composite materials that are to be developed for this application. Biological techniques have been set in place to facilitate the 
assaying of favorability of the cell response to the given materials in vitro, prior to their use in animal or clinical studies, yet a lot of work to conquer significant challenges lies ahead. For example, the rapid reorganization of the surface layers of ionic compounds in aqueous media disables their functionalization with therapeutic ligands that would be of a comparable level of stability as that achievable using silanol, thiol and phos-phonic acid moieties on the surfaces of silica, gold and quantum dot nanoparticles, respectively. To overcome this issue, however, exciting composites with versatile surface compositions, structures and functional synergies have been created, proving that problems are indeed the mothers of invention.

An essential issue that needs to be mentioned pertains to the fundamental unreliability of in vitro studies per se. For example, the same vitamin D derivative produced thoroughly different effects on differentiation and mineralization in two different osteoblast-like cultures: catabolic in one and anabolic in another [86]. Or, whereas mild cytotoxicity can wipe out cells cultured in dishes, it can provide conditions for an optimal inflammatory response that stimulates a cascade of regenerative processes in tissues [87-89]. Then, as elaborated earlier [90], drug release profiles observed in vitro and often mistakenly used as data to accompany in vivo observations are subject to change depending on the release conditions (ionic strength and $\mathrm{pH}$ of the medium, its volume, temperature, replenishment frequency, etc.), while any attempts to standardize their measurements would be meaningless in view of the fact that any two given points in the body will have different release environments. Next, there is a question of the commonly observed discrepancy between the performance of a drug delivery technology in vitro and in vivo. A higher concentration of enzymes, free radicals, antibodies and other protein species adsorbed on the surface of the carriers could all significantly accelerate its degradation and increase the kinetic order of the release profiles. These factors, in addition to phagocytosis and increased wetting, are responsible for the fact that PLGA scaffolds, for instance, degrade faster in vivo than in vitro [91]. We know now that although size, shape, surface charge, chemistry and mechanical properties of nanoparticles influence their biodistribution profiles, the route of uptake and the mechanism of interaction with the cell are mainly determined by their protein corona [92], and similar effects are to be expected with respect to the cell and tissue response to any material in a biological milieu. And whether the degradation and release will be hindered or augmented greatly depends not only on the environment, but on the type of the material too: thus, while the degradation of aliphatic polyesters is slowed down under intense fluid flows due to dissipation of acidic byproducts that would have sped up the degradation process, the fluid flow prevents local supersaturation/reprecipitation and accelerates the degradation of alkaline calcium phosphates. On the other hand, although in vivo analyses may yield more relevant insights as to how large or low the applicative potential of given therapeutic platforms at the clinical level would be, the failure of drug delivery devices and other implants for non-load-bearing applications begins from the tissue/ device interface and in vitro studies present expedient models for probing this interaction at the basic levels, including cellular, biomolecular and quantum chemistry ones. Still, how to devise in vitro drug release testing procedures that would be able to replicate in vivo conditions better, typically characterized by: i) a more dynamic flow of fluids; ii) specific local $\mathrm{pH}$ profiles that are often disease-dependent; and iii) much more complex and selective 
media, currently stands forth as a grand challenge before the drug delivery field. Complex bioreactors for mimicking biological processes in need of better understanding [93] or stimulating differentiation and assembly of stem cells [94] have been designed under the conditions of continuous flow and using the same approach to create microenvironments for more biologically relevant testing of release properties in vitro proves as a necessity from the perspective of ensuring smoother transitions from the proof-of-concept stage to commercial and clinically applicable drug delivery vehicles.

There are, of course, many other systemic challenges that define the future of the field. For example, as reports on synergetic effects between the components of drug delivery vehicles multiply $[95,96]$, it is time to reconsider the separation of drug discoverers from drug deliverers, who have traditionally worked in virtual isolation from each other. With the requirements to think fast and find reliable alternatives to antibiotics, which currently have a hard time navigating between Scylla of the long regulatory path and Charybdis of the rising microbial resistance, the need for the intercrossing of knowledge of materials scientists, drug delivery experts and pharmacologists can hardly be overstated. In the end, with the rising demands for therapeutic, regenerative and controlled delivery aspects of medical devices to be crafted in synchrony, such broadening of views and tying specific experimental approaches within ample multidisciplinary networks could be seen as all but evitable.

This is all to say that the search for a perfect drug delivery carrier for the treatment of bone dysfunction continues. Meanwhile, the room for design appears limitless and the future for the field of nanoparticulate drug delivery carriers is certain in one sense only: it belongs to multifunctional, synergistic and theranostic composite carriers capable of simultaneously targeting, monitoring and treating internal organismic disturbances in a smart, feedback fashion and in direct response to the demands of the local microenvironment and beyond.

\section{Acknowledgments}

Writing of this review article was supported by the National Institute of Health grant R00-DE021416: Osteogenic calcium phosphate nanoparticles with designable drug release kinetics.

\section{Bibliography}

Papers of special note have been highlighted as either of interest $(\bullet)$ or of considerable interest $(\bullet)$ to readers.

1. Estimates of funding for various research, condition, and disease categories (RCDC). U.S. Department of Health and Human Services; 2013. Available from: http://report.nih.gov/ categorical_spending.aspx

2•. Senneville E, Nguyen S. Current pharmacotherapy options for osteomyelitis: convergences, divergences and lessons to be drawn. Expert Opin Pharmacother. 2013; 14:723-34. An opinion piece describing microbiological etiology of osteomyelitis and explicating concerns regarding the systemic antibiotic treatment of the disease. [PubMed: 23496344]

3•. Hatzenbuehler J, Pulling TJ. Diagnosis and management of osteomyelitis. Am Fam Physician. $2011 ; 84: 1027-33$. A brief piece outlining the basics of etiology, of clinical features, of diagnostic imaging, and of the treatment of osteomyelitis. [PubMed: 22046943]

4••. Lew DP, Waldvogel FA. Osteomyelitis. Lancet. 2004; 364:369-79. A review article on clinical features of osteomyelitis, classification of the disease depending on the symptoms and the origin, and traditional medical management thereof. [PubMed: 15276398] 
5. Moodie, RL. An introduction to the ancient evidences of disease. University of Illinois Press; Chicago: 1923. p. 243-82.

6. Klenerman L. A history of osteomyelitis from the Journal of Bone and Joint Surgery: 1948 to 2006. J Bone Joint Surg Br. 2007; 89:667-70. [PubMed: 17540756]

7. Orr HW. The treatment of acute osteomyelitis by drainage and rest. Clin Orthop Relat Res. 1927; 9:733-9.

8. Baer WS. The classic: the treatment of chronic osteomyelitis with the maggot (larva of the blow fly) 1931. Clin Orthop Relat Res. 2011; 469:920-44. [PubMed: 20532712]

9••. Buchholz HW, Engelbrecht H. Depot effects of various antibiotics mixed with Palacos resins. Chirurg. 1970; 41:511-15. The first paper to report a clinical application of poly(methyl methacrylate) PMMA beads for the local delivery of antibiotics in the treatment of osteomyelitis. [PubMed: 5487941]

10. Giavaresi G, Borsari V, Fini M, et al. Preliminary investigations on a new gentamicin and vancomycin-coated PMMA nail for the treatment of bone and intramedullary infections: an experimental study in the rabbit. J Orthop Res. 2008; 26:785-92. [PubMed: 18186130]

11. Roeder B, Van Gils CC, Maling S. Antibiotic beads in the treatment of diabetic pedal osteomyelitis. J Foot Ankle Surg. 2000; 39:124-30. [PubMed: 10789104]

12. Nelson CL, Hickmon SG, Harrison BH. Elution characteristics of gentamicin- PMMA beads after implantation in humans. Orthopedics. 1994; 17:415-16. [PubMed: 8036185]

13•. Kluin OS, van der Mei HC, Busscher HJ, Neut D. Biodegradable vs non-biodegradable antibiotic delivery devices in the treatment of osteomyelitis. Expert Opin Drug Deliv. 2013; 10:341-51. A review piece highlighting the drawbacks of non-biodegradable antibiotic carriers in the treatment of osteomyelitis and proposing a wider use of biodegradable alternatives. [PubMed: 23289645]

14. Moojen DJ, Hentenaar B, Charles Vogely H, et al. In vitro release of antibiotics from commercial PMMA beads and articulating hip spacers. J Arthroplasty. 2008; 23:1152-6. [PubMed: 18534493]

15. Wong MW, Hui M. Development of gentamicin resistance after gentamicin-PMMA beads for treatment of foot osteomyelitis: report of two cases. Foot Ankle Int. 2005; 26:1093-5. [PubMed: 16390644]

16•. Anagnostakos K, Hitzler P, Pape D, et al. Persistence of bacterial growth on antibiotic-loaded beads: is it actually a problem? Acta Orthop. 2008; 79:302-7. A paper reporting on the persistence of bacterial growth on gentamicin- and vancomycin-loaded PMMA beads. [PubMed: 18484259]

17. Bereznowski Z. In vivo assessment of methyl methacrylate metabolism and toxicity. Int J Biochem Cell Biol. 1995; 27:1311-16. [PubMed: 8581827]

18•. Barth RE, Vogely HC, Hoepelman AI, Peters EJ. 'To bead or not to bead?' Treatment of osteomyelitis and prosthetic joint-associated infections with gentamicin bead chains. Int $\mathbf{J}$ Antimicrob Agents. 2011; 38:371-5. A short opinion piece succinctly highlighting the cons of gentamicin-loaded PMMA bead chains in treating osteomyelitis. [PubMed: 21549570]

19. Jepegnanam TS, von Schroeder HP. Rapid resorption of calcium sulfate and hardware failure following corrective radius osteotomy: 2 case reports. J Hand Surg Am. 2012; 37:477-80. [PubMed: 22305728]

20. Ziran BH, Smith WR, Morgan SJ. Use of calcium-based demineralized bone matrix/allograft for nonunions and posttraumatic reconstruction of the appendicular skeleton: preliminary results and complications. J Trauma. 2007; 63:1324-8. [PubMed: 18212656]

21. Robinson D, Alk D, Sandbank J, et al. Inflammatory reactions associated with a calcium sulfate bone substitute. Ann Transplant. 1999; 4(3-4):91-7. [PubMed: 10853791]

22. von Rechenberg B, Génot OR, Nuss K, et al. Evaluation of four biodegradable, injectable bone cements in an experimental drill hole model in sheep. Eur J Pharm Biopharm. 2013; 85:130-8. [PubMed: 23680585]

23. Wei G, Kotoura Y, Oka M, et al. A bioabsorbable delivery system for antibiotic treatment of osteomyelitis: the use of lactic acid oligomer as a carrier. J Bone Joint Surg Br. 1991; 73(2):24652. [PubMed: 2005148]

24. Garvin K, Feschuk C. Polylactide-polyglycolide antibiotic implants. Clin Orthop Relat Res. 2005; 437:105-10. [PubMed: 16056034] 
25. Koort JK, Makinen TJ, Suokas E, et al. Sustained release of ciprofloxacin from an osteoconductive poly(DL)-lactide implant. Acta Orthop. 2008; 79:295-301. [PubMed: 18484258]

26. Danhier F, Ansorena E, Silva JM, et al. PLGA-based nanoparticles: an overview of biomedical applications. J Control Release. 2012; 161:505-22. [PubMed: 22353619]

27. Stevanović M, Savić J, Jordović B, Uskoković D. Fabrication, in vitro degradation and the release behaviours of poly(DL-lactide-co-glycolide) nanospheres containing ascorbic acid. Colloids Surf B Biointerfaces. 2007; 59:215-23. [PubMed: 17596919]

28. Mistry AS, Mikos AG. Tissue engineering strategies for bone regeneration. Adv Biochem Eng Biotechnol. 2005; 94:1-22. A review article spotlighting limitations of the current clinical treatments for bone defects and the most prospective alternatives in the research and clinical trial phases. [PubMed: 15915866]

29. Bostman OM. Osteotic changes accompanying degradation of absorbable fracture fixation implants. J Bone Joint Surg Br. 1991; 73:679-82. [PubMed: 1649195]

30. Bostman O, Hirvensalo E, Mikinen J, Rokkanen P. Foreign-body reaction to fracture fixation implants of biodegradable synthetic polymers. J Bone Joint Surg Br. 1990; 72:592-6. [PubMed: 2199452]

31. Solheim E, Sudmann B, Bang G, Sudmann E. Biocompatibility and effect on osteogenesis of poly(ortho ester) compared to poly(DL-lactic acid). J Biomed Mater Res. 2000; 49:257-63. [PubMed: 10571914]

32. Tiainen J, Soini Y, Suokas E, et al. Tissue reactions to bioabsorbable ciprofloxacin-releasing polylactide-polyglycolide 80/20 screws in rabbits' cranial bone. J Mater Sci Mater Med. 2006; 17:1315-22. [PubMed: 17143763]

33. Plachokova A, Link D, van den Dolder J, et al. Bone regenerative properties of injectable PGLACaP composite with TGF-beta1 in a rat augmentation model. J Tissue Eng Regen Med. 2007; 1:457-64. [PubMed: 18265419]

34. Lemaire S, Van Bambeke F, Pierard D, et al. Activity of fusidic acid against extracellular and intracellular Staphylococcus aureus: influence of $\mathrm{pH}$ and comparison with linezolid and clindamycin. Clin Infect Dis. 2011; 52:S493-503. [PubMed: 21546626]

$35 \bullet$. Uskoković V, Uskoković DP. Nanosized hydroxyapatite and other calcium phosphates: chemistry of formation and application as drug and gene delivery agents. J Biomed Mater Res B Appl Biomater. 2011; 96(1):152-91. A comprehensive review piece discussing the chemistry of formation and postsynthetic transformations of calcium phosphates and their potential use as ubiquitous drug delivery carriers. [PubMed: 21061364]

36. Suen RB, Lin SC, Hsu WH. Hydroxyapatite-based immobilized metal affinity adsorbents for protein purification. J Chromatogr A. 2004; 1048:31-9. [PubMed: 15453416]

37. Pinto G, Caira S, Cuollo M, et al. Hydroxyapatite as a concentrating probe for phosphoproteomic analyses. J Chromatogr B Analyt Technol Biomed Life Sci. 2010; 878:2669-78.

38. Kazemzadeh-Narbat M, Noordin S, Masri BA, et al. Drug release and bone growth studies of antimicrobial peptide-loaded calcium phosphate coating on titanium. J Biomed Mater Res B Appl Biomater. 2012; 100:1344-52. [PubMed: 22566395]

39. Petrone C, Hall G, Langman M, Filiaggi MJ. Compaction strategies for modifying the drug delivery capabilities of gelled calcium polyphosphate matrices. Acta Biomater. 2008; 4:403-13. [PubMed: 17997374]

40. Rauschmann MA, Wichelhaus TA, Stirnal V, et al. Nanocrystalline hydroxyapatite and calcium sulphate as biodegradable composite carrier material for local delivery of antibiotics in bone infections. Biomaterials. 2005; 26:2677-84. [PubMed: 15585271]

41. Itokazu M, Yang W, Aoki T, et al. Synthesis of antibiotic-loaded interporous hydroxyapatite blocks by vacuum method and in vitro drug release testing. Biomaterials. 1998; 19:817-19. [PubMed: 9663758]

42. Uskoković V, Košak A, Drofenik M. Preparation of silica-coated lanthanum-strontium manganite particles with designable Curie point, for application in hyperthermia treatments. Int J Appl Ceram Tech. 2006; 3:134-43.

43. Uskoković V, Desai TA. Phase composition control of calcium phosphate nanoparticles for tunable drug delivery kinetics and treatment of osteomyelitis. Part 1: preparation and drug 
release. J Biomed Mater Res A. 2013; 101:1416-26. A study demonstrating that stoichiometry of calcium phosphates could be used to control their degradability and, thus, the antibiotic release rate, which could be therefore tuned to match a desired release profile. [PubMed: 23115118]

44. Rahaman MN, Bal BS, Huang W. Review: emerging developments in the use of bioactive glasses for treating infected prosthetic joints. Mater Sci Eng C Mater Biol Appl. 2014; 41C:224-31. [PubMed: 24907755]

45. Fu Q, Rahaman MN, Fu H, Liu X. Silicate, borosilicate, and borate bioactive glass scaffolds with controllable degradation rate for bone tissue engineering applications. I. preparation and in vitro degradation. J Biomed Mater Res A. 2010; 95(1):164-71. [PubMed: 20544804]

46. Ni S, Du R, Ni S. The Influence of $\mathrm{Na}$ and $\mathrm{Ti}$ on the in vitro degradation and bioactivity in $58 \mathrm{~S}$ Sol-Gel bioactive glass. Adv Mat Sci Eng. 2012; 2012:730810.

47. Fredholm YC, Karpukhina N, Brauer DS, et al. Influence of strontium for calcium substitution in bioactive glasses on degradation, ion release and apatite formation. J R Soc Interface. 2012; 9:880-9. [PubMed: 21993007]

48. Jugdaohsingh R. Silicon and bone health. J Nutr Health Aging. 2007; 11(2):99-110. [PubMed: 17435952]

49. Rahaman MN, Day DE, Bal BS, et al. Bioactive glass in tissue engineering. Acta Biomater. 2011; 7:2355-73. [PubMed: 21421084]

50. Confavreux CB. Bone: from a reservoir of minerals to a regulator of energy metabolism. Kidney Int Suppl. 2011; 121:S14-19. A review piece describing the multifunctional nature of bone, including the mechanistic basics of energy metabolism. [PubMed: 21346725]

51. Park EK, Lee YE, Choi JY, et al. Cellular biocompatibility and stimulatory effects of calcium metaphosphate on osteoblastic differentiation of human bone marrow-derived stromal cells. Biomaterials. 2004; 25:3403-11. [PubMed: 15020113]

52. Liu YK, Lu QZ, Pei R, et al. The effect of extracellular calcium and inorganic phosphate on the growth and osteogenic differentiation of mesenchymal stem cells in vitro: implication for bone tissue engineering. Biomed Mater. 2009; 4:025004. [PubMed: 19208939]

53. Mailland M, Waelchli R, Ruat M, et al. Stimulation of cell proliferation by calcium and a calcimimetic compound. Endocrinology. 1997; 138:3601-5. [PubMed: 9275041]

54••. Dorozhkin S. Self-setting calcium orthophosphate formulations. J Funct Biomater. 2013; 4:209_ 311. A comprehensive review of self-setting calcium orthophosphates, describing the basic mechanism of the process, classification based on the final product, some practical issues, and the types of commercial mixtures available. [PubMed: 24956191]

55. Uskoković V, Batarni SS, Schweicher J, et al. The effect of morphology of calcium phosphate nanoparticles for sustained release of antibiotics on their antibacterial and osteogenic performance. ACS Appl Mater Interfaces. 2013; 5:2422-31. [PubMed: 23484624]

56•. Uskoković V. Prospects and pits on the path of biomimetics: the case of tooth enamel. J Biomim Biomater Tissue Eng. 2010; 8:45-78. A review piece questioning unquestioned prospects of biomimetic strategies in the design of advanced materials in general and hard tissue substitutes in particular.

57. Gebeshuber IC, Gruber P, Drack M. A gaze into the crystal ball - biomimetics in the year 2059. Proc IMechE Part C: J Mech Eng Sci. 2009; 223:2899-918.

58. Ziv V, Weiner S. Bone crystal sizes: a comparison of transmission electron microscopic and X-ray diffraction line width broadening techniques. Connect Tissue Res. 1993; 29:1-11. [PubMed: 8339541]

59. Saito H, Takeda S, Amizuka N. Eldecalcitol and calcitriol stimulates 'bone minimodeling,' focal bone formation without prior bone resorption, in rat trabecular bone. J Steroid Biochem Mol Biol. 2013; 136:178-82. [PubMed: 23069645]

60. Lie KK, Moren M. Retinoic acid induces two osteocalcin isoforms and inhibits markers of osteoclast activity in Atlantic cod (Gadus morhua) ex vivo cultured craniofacial tissues. Comp Biochem Physiol A Mol Integr Physiol. 2012; 161:174-84. [PubMed: 22075542]

61. Lind T, Sundqvist A, Hu L, et al. Vitamin A is a negative regulator of osteoblast mineralization. PLoS One. 2013; 8:e82388. [PubMed: 24340023] 
62. Strathmann FG, Laha TJ, Hoofnagle AN. Quantification of 1alpha,25-dihydroxy vitamin D by immunoextraction and liquid chromatography-tandem mass spectrometry. Clin Chem. 2011; 57:1279-85. [PubMed: 21768219]

63. Lisse TS, Chun RF, Rieger S, et al. Vitamin D activation of functionally distinct regulatory miRNAs in primary human osteoblasts. J Bone Miner Res. 2013; 28:1478-88. [PubMed: 23362149]

64- Hudson MC, Ramp WK, Nicholson NC, et al. Internalization of Staphylococcus aureus by cultured osteoblasts. Microb Pathog. 1995; 19(6):409-19. Although the ability of S. aureus to enter eukaryotic cells had been documented earlier, this is the first report on their internalization by osteoblasts as well as on a temporary coexistence of bacterial colonies and the host cells. [PubMed: 8852281]

65••. Koziel J, Maciag-Gudowska A, Mikolajczyk T, et al. Phagocytosis of Staphylococcus aureus by macrophages exerts cytoprotective effects manifested by the upregulation of antiapoptotic factors. PLoS One. 2008; 4:e5210. A research report demonstrating a protective effect on macrophages infected by $\mathrm{S}$. aureus against cell death induced by the apoptotic initiator, staurosporine. [PubMed: 19381294]

66. Bayles KW, Wesson CA, Liou LW, et al. Intracellular Staphylococcus aureus escapes the endosome and induces apoptosis in epithelial cells. Infect Immun. 1998; 66:336-42. [PubMed: 9423876]

67. Ellington JK, Harris M, Webb L, et al. Intracellular staphylococcus aures: a mechanism for the indolence of osteomyelitis. J Bone Joint Surg Br. 2003; 85:918-21. [PubMed: 12931819]

68. Hassan MA, Ahmed IS, Campbell P, Kondo T. Enhanced gene transfection using calcium phosphate co-precipitates and low-intensity pulsed ultrasound. Eur J Pharm Sci. 2012; 47:768-73. [PubMed: 22921720]

69. Elangovan S, Jain S, Tsai PC, et al. Nano-sized calcium phosphate particles for periodontal gene therapy. J Periodontol. 2013; 84:117-25. [PubMed: 22414259]

70•. Uskoković V, Desai TA. Simultaneous bactericidal and osteogenic effect of nanoparticulate calcium phosphate powders loaded with clindamycin on osteoblasts onfected with Staphylococcus aureus. Mater Sci Eng C Mater Biol Appl. 2014; 37:210-22. A research report showing that clindamycin-loaded calcium phosphates could be more effective in decreasing the population of $\mathrm{S}$ aureus colonizing osteoblasts and slowing down bacterial proliferation than the pure antibiotic. [PubMed: 24582242]

71. Uskoković V, Hoover C, Vukomanović M, et al. Osteogenic and antimicrobial nanoparticulate calcium phosphate and/or poly-lactide-co-glycolide powders for the treatment of osteomyelitis. Mater Sci Eng C Mater Biol Appl. 2013; 33:3362-73. [PubMed: 23706222]

72•. Jain AK, Das M, Swarnakar NK, Jain S. Engineered PLGA nanoparticles: an emerging delivery tool in cancer therapeutics. Crit Rev Ther Drug Carrier Syst. 2011; 28:1-45. A paper listing the potential applications for surface-grafted poly-DL-lactide-co-glycolide in the controlled delivery of chemotherapeutic agents. [PubMed: 21395514]

73. Xu Q, Czernuszka JT. Controlled release of amoxicillin from hydroxyapatite-coated poly(lactic-coglycolic acid) microspheres. J Control Release. 2008; 127:146-53. [PubMed: 18325617]

74. Naito K, Obayashi O, Mogami A, et al. Fracture of the calcium phosphate bone cement which used to enchondroma of the hand, a case report. Eur J Orthop Surg Traumatol. 2008; 18:405-8.

75•. Rezwan K, Chen Q, Blaker J, Boccaccini A. Biodegradable and bioactive porous polymer/ inorganic composite scaffolds for bone tissue engineering. Biomaterials. 2006; 27:3413-31. A review of materials and fabrication routes for three-dimensional scaffolds for bone tissue engineering. [PubMed: 16504284]

76. Bucholz RW. Nonallograft osteoconductive bone graft substitutes. Clin Orthop Relat Res. 2002; 395:44-52. [PubMed: 11937865]

77. Ajduković Z, Ignjatović N, Petrović D, Uskoković D. Substitution of osteoporotic alveolar bone by biphasic calcium phosphate/poly-DL-lactide-co-glycolide biomaterials. J Biomater Appl. 2007; 21:317-28. [PubMed: 17229816] 
78. Ruhè PQ, Hedberg EL, Padron NT, et al. Biocompatibility and degradation of poly(DL-lactic-coglycolic acid)/calcium phosphate cement composites. J Biomed Mater Res A. 2005; 74:533-44. [PubMed: 16041795]

79. Félix Lanao RP, Leeuwenburgh SC, Wolke JG, Jansen JA. Bone response to fast-degrading, injectable calcium phosphate cements containing PLGA microparticles. Biomaterials. 2011; 32:8839-47. [PubMed: 21871661]

80. Nehilla BJ, Allen PG, Desai TA. Surfactant-free, drug-quantum-dot coloaded poly(lactide-coglycolide) nanoparticles: towards multifunctional nanoparticles. ACS Nano. 2008; 2:528-44.

81. Guo Y, Shi DL, Lian J, et al. Quantum dot conjugated hydroxylapatite nanoparticles for in vivo imaging. Nanotechnology. 2008; 19:175102. [PubMed: 21825660]

82-. Stepensky D, Kleinberg L, Hoffman A. Bone as an effect compartment: models for uptake and release of drugs. Clin Pharmacokinet. 2003; 42:863-81. A review covering pharmacokinetic and pharmacodynamic features of an array of bone-targeting agents. [PubMed: 12885262]

83. Redini F, Odri GA, Picarda G, et al. Drugs targeting the bone microenvironment: new therapeutic tools in Ewing's sarcoma? Expert Opin Emerg Drugs. 2013; 18:339-52. [PubMed: 23957761]

84. Takai H, Matsumura H, Matsui S, et al. Unliganded estrogen receptor a stimulates bone sialoprotein gene expression. Gene. 2014; 539:50-7. [PubMed: 24495337]

85. Hou T, Xu J, Li Q, et al. In vitro evaluation of a fibrin gel antibiotic delivery system containing mesenchymal stem cells and vancomycin alginate beads for treating bone infections and facilitating bone formation. Tissue Eng Part A. 2008; 14:1173-82. [PubMed: 18593356]

86. Yang D, Atkins GJ, Turner AG, et al. Differential effects of 1,25-dihydroxyvitamin D on mineralisation and differentiation in two different types of osteoblast-like cultures. J Steroid Biochem Mol Biol. 2013; 136:166-70. [PubMed: 23220547]

87. Ignjatović N, Ajduković Z, Savić V, et al. Nanoparticles of cobalt-substituted hydroxyapatite in regeneration of mandibular osteoporotic bones. J Mater Sci Mater Med. 2013; 24:343-54. [PubMed: 23090835]

88• Ignjatović N, Uskoković V, Ajduković Z, Uskoković D. Multifunctional hydroxyapatite and poly(D,L-lactide-co-glycolide) nanoparticles for the local delivery of cholecalciferol. Mater Sci Eng C Mater Bio Appl. 2013; 33:943-50. A research study demonstrating that the apoptosis of osteoblastic cells caused by delivering vitamin $\mathrm{D}_{3}$ thereto directly from the surface of hydroxyapatite nanoparticles can be prevented by coating them with poly (D,L-lactide-coglycolide) and thus ensuring a more sustained release profile. [PubMed: 25382938]

89. Wu X, Wang W, Meng C, et al. Regulation of differentiation in trabecular bone-derived mesenchymal stem cells by T cell activation and inflammation. Oncol Rep. 2013; 30:2211-19. [PubMed: 23970332]

90••. Uskoković V. Entering the era of nanoscience: time to be so small. J Biomed Nanotechnol. 2013; 9:1441-70. A discourse on some of the main challenges faced by research at the frontier of nanoscience. [PubMed: 23980495]

91. Oh SH, Kang SG, Lee JH. Degradation behaviour of hydrophilized PLGA scaffolds prepared by melt-moulding particulate-leaching method: comparison with control hydrophobic one. J Mater Sci Mater Med. 2006; 17:131-7. [PubMed: 16502245]

92-. Saptarshi SR, Duschl A, Lopata AL. Interaction of nanoparticles with proteins: relation to bioreactivity of the nanoparticle. J Nanobiotechnology. 2013; 11:26. Description of nanoparticleprotein corona complexes forming upon the release of nanoparticles into any biological environment. [PubMed: 23870291]

93••. Uskoković V, Li W, Habelitz S. Amelogenin as a promoter of nucleation and crystal growth of apatite. J Crystal Growth. 2011; 316:106-17. A study that reverted the paradigm regarding the role of the main protein of the developing enamel matrix in guiding the formation of this tooth tissue.

94-. Burdick JA, Vunjak-Novaković G. Engineered microenvironments for controlled stem cell differentiation. Tissue Eng Part A. 2009; 15:205-19. A review discussing bioreactors as dynamic three-dimensional environments guiding the fate and controlling the function of stem cells. [PubMed: 18694293] 
95. Chaowanachan T, Krogstad E, Ball C, Woodrow KA. Drug synergy of tenofovir and nanoparticlebased antiretrovirals for HIV prophylaxis. PLoS One. 2013; 8:e61416. [PubMed: 23630586]

96. Seidlits SK, Gower RM, Shepard JA, Shea LD. Hydrogels for lentiviral gene delivery. Expert Opin Drug Deliv. 2013; 10:499-509. [PubMed: 23347508]

97. Kim JJ, Bae WJ, Kim JM, et al. Mineralized polycaprolactone nanofibrous matrix for odontogenesis of human dental pulp cells. J Biomater Appl. 2014; 28(7):1069-78. [PubMed: 23839784]

98. Zhao F, Zhao Y, Liu Y, et al. Cellular uptake, intracellular trafficking, and cytotoxicity of nanomaterials. Small. 2011; 7:1322-37. [PubMed: 21520409]

99. Stevanović M, Savanović I, Uskoković V, et al. A new, simple, green and one-pot four-component synthesis of bare and poly(alpha, gamma, L-glutamic acid) capped silver nanoparticles. Colloid Polymer Sci. 2011; 290:221-31.

100••. Stevanović M, Uskoković V, Filipović M, et al. Composite PLGA/AgNpPGA/AscH nanospheres with combined osteoinductive, antioxidative and antimicrobial activities. ACS Appl Mat Interfaces. 2013; 5:9034-42. A research piece describing the fabrication of a multifunctional composite nanomaterial composed of poly(L-glutamic acid)-capped silver nanoparticles coated with a layer of poly(lactide-co-glycolide) (PLGA) loaded with ascorbic acid, combining osteogenic, antimicrobial and antioxidative properties in one. 


\section{Article highlights}

- A great demand exists for the development of antimicrobial bone grafts.

- Similia similibus curantur principle dictates that calcium phosphate should be the key inorganic ingredient of composites for bone regeneration.

- Biodegradable poly(a-hydroxy esters) are common compounds of choice for the organic ingredient of composites for bone regeneration, but their shortcomings are many and are discussed herein.

- Staphylococcus aureus, the main causative agent of osteomyelitis, is able to penetrate bone cells and form intracellular colonies, so that design of antibiotic therapies that target them may be more relevant for treating chronic bone infection than eliminating only the pathogens colonizing the bone matrix.

- The problem of disparity between in vitro drug release testing procedures and in vivo conditions that they mimic is discussed.

- It is concluded that ideal implants or injects for orthopedic infection therapies would be multifunctional composites capable of simultaneously targeting and treating the disease from two angles: antimicrobial and osteogenic.

This box summarizes key points contained in the article. 


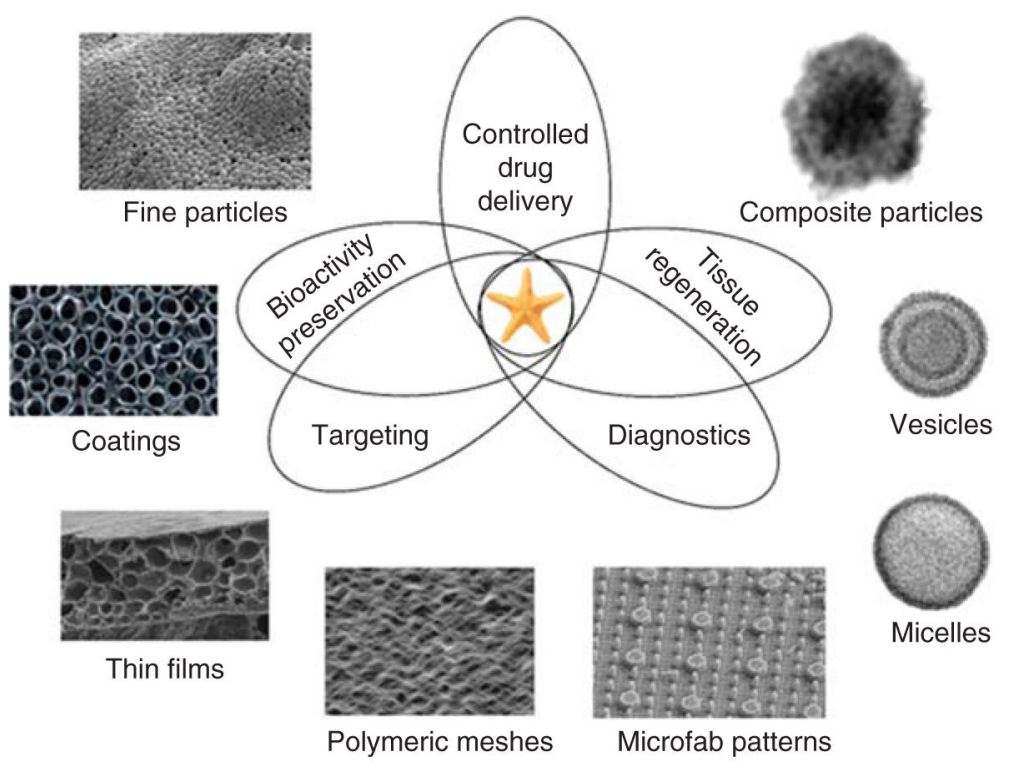

Figure 1. There is an increasing demand for contemporary drug delivery platforms to be multifunctional, that is, to simultaneously: i) deliver the drug smartly, at the optimal rate and amount over prolonged periods of time and in feedback with the characteristics of the local microenvironment; ii) preserve the drug's bioactivity in the biological milieu; iii) contribute to tissue regeneration; iv) promote targeting of the right location at the cellular or subcellular level; and $v$ ) act as traceable sensors to serve a diagnostic purpose in real time

Some of the sophisticated material structures intensely researched for such applications include soft, hard and composite nano- and microparticles, thin films and surface coatings, organic meshes, micelles and vesicles, and microfabricated patterns. 
A.

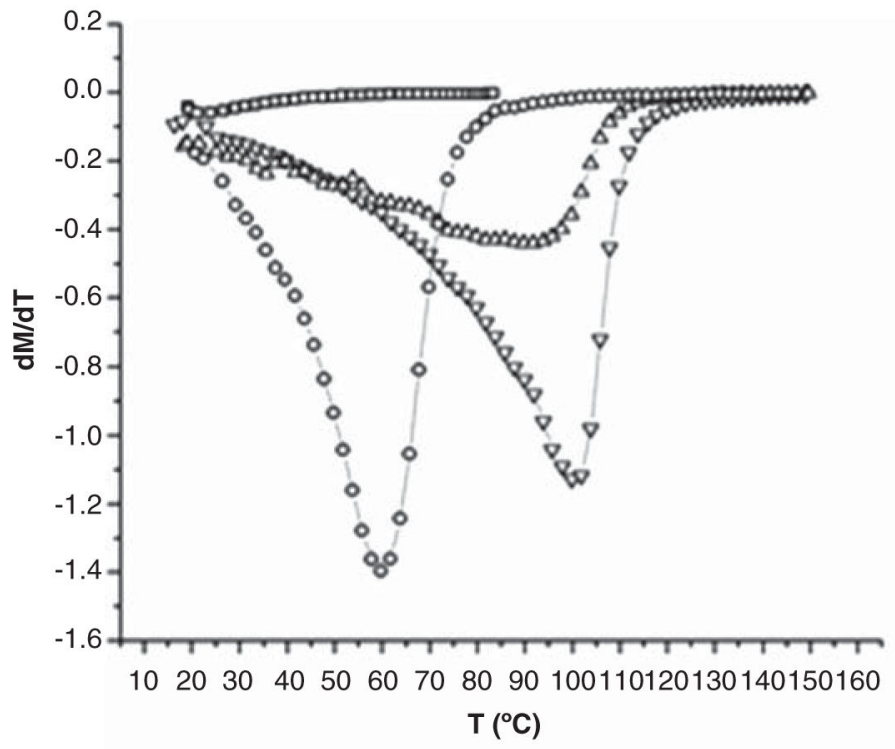

B.

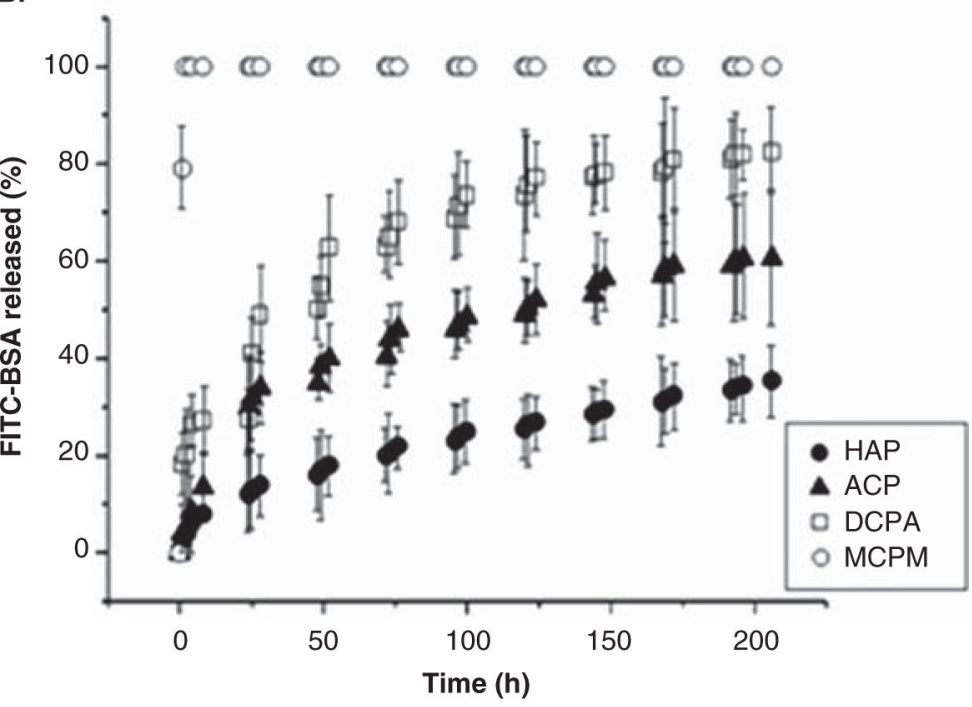

Figure 2.

(A) The derivative of specific magnetization over time as a function of temperature for powders comprising nanosized $\mathrm{La}_{1-\mathrm{x}} \mathrm{Sr}_{\mathrm{x}} \mathrm{MnO}_{3}+\delta$ particles with different values of parameter $\mathrm{x}[\mathrm{x}=0.16(\square), 0.24(\bigcirc), 0.33(\triangle)$ and $0.5(\boldsymbol{\nabla})]$, with the local minima corresponding to the Neel point of the material, the temperature at which the magnetic moments become randomized due to thermal fluctuations. (B) Different kinetic profiles for the release of albumin (FITC-BSA) from compacted nanostructured calcium phosphate powders of different phase compositions: MCPM, DCPA, ACP and HAP.

Reprinted with permission from $[42,43]$.

ACP: Amorphous calcium phosphate; DCPA: Dicalcium phosphate anhydrous; HAP:

Hydroxyapatite; MCPM: Monocalcium phosphate monohydrate. 


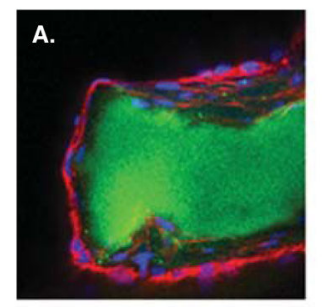

c.

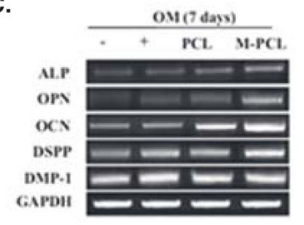

B.

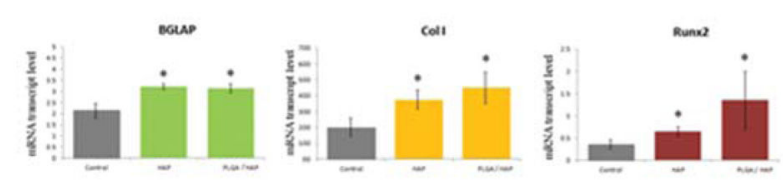

(HOB; P11)
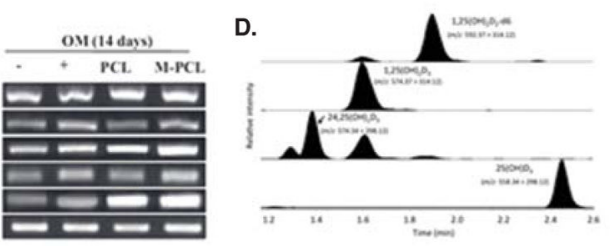

E.

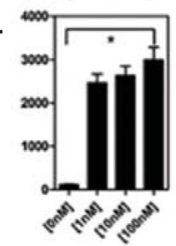

Figure 3.

Morphological (A), genetic (B), proteomic (C) and metabolic (D) assessment of the osteogenic effectiveness of nanoparticles delivering a therapeutic payload. (A)

Immunofluorescently counterstained osteoblasts (nuclei - blue; f-actin -red) are shown adhering onto a microscopic conglomerate of calcium phosphate nanoparticles (green) capable of delivering an antibiotic payload to the segments of infected bone; (B) mRNA expression of three different osteogenic markers - osteocalcin (BGLAP), protocollagen type $\mathrm{I}(\mathrm{Col} \mathrm{I})$ and the transcription factor Runx 2 - in osteoblasts seeded in the presence of hydroxyapatite/PLGA core-shell composite particles is greater than in osteoblasts seeded in the presence of pure hydroxyapatite, which is itself greater than in control osteoblasts; (C) Western blot analysis of the expression of different proteins of the osteogenic pathway in the presence of mineralized (M-PCL) and regular (PCL) poly( $\varepsilon$-caprolactone) at different time points; (D) High-performance liquid chromatograms showing peaks of cholecalciferol metabolites capable of increasing the concentration of osteocalcin secreted by osteoblasts (E).

Reprinted with permissions from [71] and [97].

PLGA: Poly-(D,L-lactide-co-glycolide). 
A.

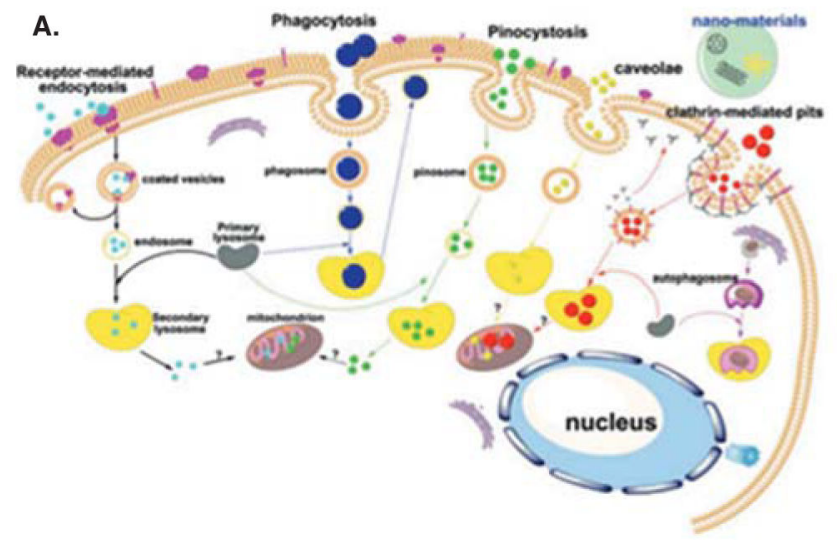

B.

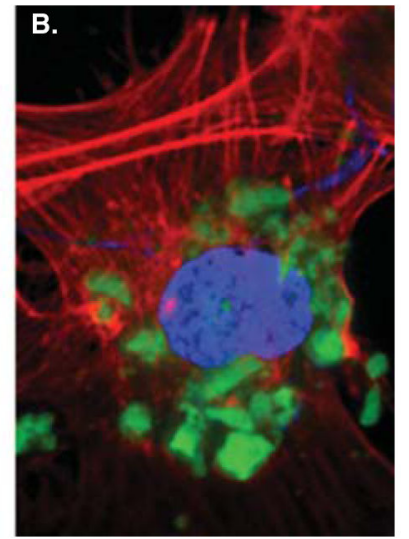

Figure 4.

(A) A schematic description of the major pathways for the intracellular uptake of nanoparticles. (B) A single-plane confocal optical image showing the localization of aggregates of clindamycin-containing calcium phosphate nanoparticles (green) inside an osteoblastic cell (nucleus - blue; cytoskeletal f-actin - red).

Reprinted with permission from [98]. 

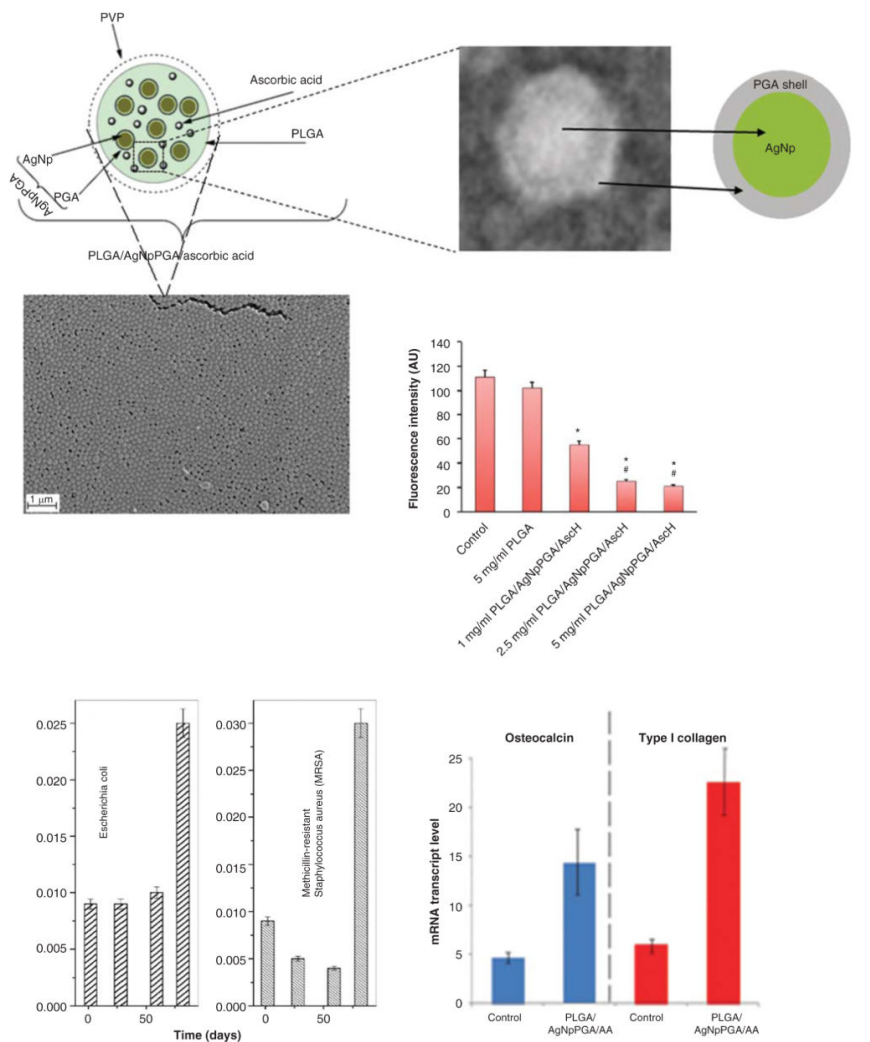

Figure 5. Composite monodisperse nanoparticles with simultaneous antioxidative, antibacterial and osteoinductive properties made by coating PLGA around silver-PGA core-shell Np and dispersing ascorbic acid inside them

Adapted and reprinted with permission from [99,100].

Np: Nanoparticles; PGA: Poly(glycolide); PLGA: Poly-(D,L-lactide-co-glycolide). 